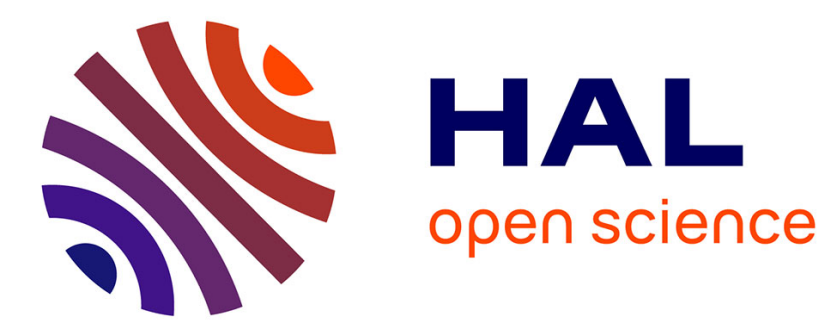

\title{
Une tombe barbare du Ve siècle à Hochfelden (Bas-Rhin) \\ Jean-Jacques Hatt
}

\section{To cite this version:}

Jean-Jacques Hatt. Une tombe barbare du Ve siècle à Hochfelden (Bas-Rhin). Gallia - Fouilles et monuments archéologiques en France métropolitaine, 1965, 23 (2), pp.250-256. 10.3406/galia.1965.2415 . hal-01933687

\section{HAL Id: hal-01933687 \\ https://hal.science/hal-01933687}

Submitted on 26 Feb 2020

HAL is a multi-disciplinary open access archive for the deposit and dissemination of scientific research documents, whether they are published or not. The documents may come from teaching and research institutions in France or abroad, or from public or private research centers.
L'archive ouverte pluridisciplinaire HAL, est destinée au dépôt et à la diffusion de documents scientifiques de niveau recherche, publiés ou non, émanant des établissements d'enseignement et de recherche français ou étrangers, des laboratoires publics ou privés.

\section{(ㅇ)(1) $\$$}

Distributed under a Creative Commons Attribution - NonCommercial - NoDerivatives| 4.0 


\section{Une tombe barbare du Ve siècle à Hochfelden (Bas-Rhin)}

Le 2 novembre 1964, une pelle mécanique conduite par un ouvrier de la tuilerie Lanter, à Hochfelden, entama une tombe située à $1,80 \mathrm{~m}$. de profondeur dans la parcelle $149 \mathrm{de}$ la section 14, appartenant encore à M. Knab, demeurant à Hochfelden, 12 rue des Bergers. La pelle enleva une partie du squelette : les deux pieds et le tibia gauche. L'ouvrier qui s'en était aperçu, et qui avait reçu des instructions de M. Lanter, dégagea alors la partie inférieure du corps jusqu'au bassin et, à la hauteur de la main droite, observa la présence de six plaquettes décoratives en or, qu'il remit à M. Lanter. Ce dernier prévint immédiatement le Musée Archéologique de Strasbourg. Le lendemain matin, M. H. Zum Stein, assistant du Musée, et M. E. Kern, agent technique de la Circonscription des antiquités historiques, se rendirent sur place. Ils procédèrent méthodiquement au dégagement du squelette, laissant à leur place les éléments du mobilier en les photographiant et les dessinant in silu.

Le cercueil, constitué de planches assemblées sans clous, se détachait encore nettement sous la forme d'une paroi droite verticale, dont la surface présentait une couleur marron foncé contrastant avec le ton clair du loess, en raison de la décomposition du bois. Les dimensions de ce cercueil étaient les suivantes : hauteur, $0,45 \mathrm{~m}$ à la tête, largeur, $0,45 \mathrm{~m}$ à la tête, $0,35 \mathrm{~m}$ aux pieds, longueur totale, environ 1,70 m. La longueur du corps peut être évaluée à environ $1,50 \mathrm{~m}$; il existait, entre la tête et l'extrémité du cercueil, un espace libre d'environ $0,20 \mathrm{~m}$. Sur la tête, sur le thorax et jusqu'au bassin, ont été observées les traces d'une planche large de 0,25 ) et longue d'environ $0,60 \mathrm{~m}$.

Les plaquettes décoratives étaient groupées en ligne autour des deux poignets, des deux coudes et du col. Les deux fibules étaient placées obliquement, la pointe en haut, de chaque côté du thorax, à l'emplacement du milieu des humérus. De chaque côté du crâne, à droite et à gauche, se trouvait une boucle d'oreille en or. Autour du cou, un collier en tresse d'or, pourvu de trente pendeloques coniques, n'était pas fermé, les deux parties du fermoir se trouvant à 0,10 l'une de l'autre et le crochet de fermeture ayant disparu (fig. 1 et, pour l'emplacement des plaques et des spirales, fig. 2).

Sous les vertèbres du dos se trouvait un miroir en bronze poli, dont la face était tournée vers le haut. A dix centimètres à gauche du crâne, un gobelet en verre vert légèrement irisé, dont l'ouverture était dirigée vers la tête (fig. 1).

Le corps était placé en décubitus dorsal, les bras le long du corps, la main droite placée sur le bassin. Il semble que le cadavre se soit légèrement affaissé vers la droite, comme en témoigne la position de la tête. La mâchoire inférieure était tombée sur les vertèbres du cou. Le crâne et les os sont en excellent état de conservation. Le squelette était orienté nordsud, la tête au nord (fig. 1).

La parure complète est reproduite fig. 3, où les fibules sont tête en bas.

Les boucles d'oreilles ont un diamètre de $2 \mathrm{~cm}$, et un poids de $4 \mathrm{gr}$ chacune. Elles n'étaient pas faites pour traverser le lobe de l'oreille, mais pour le pincer (fig. 4). Elles sont décorées à leur extrémité d'un polyèdre à quatorze faces losangiques ou triangulaires; il s'agit en fait de cubes dont les angles ont été limés. Ce genre de décor est courant sur les 

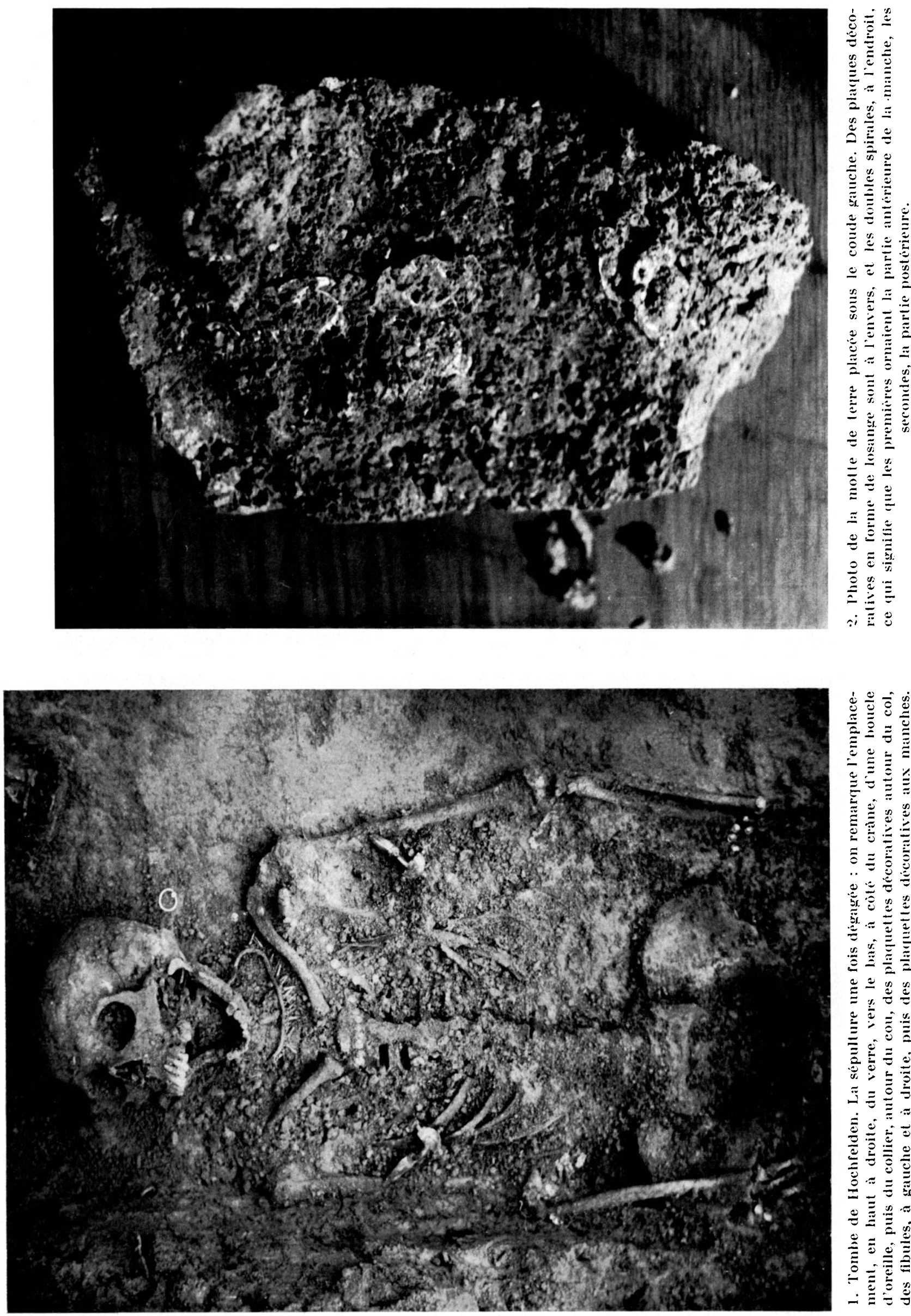

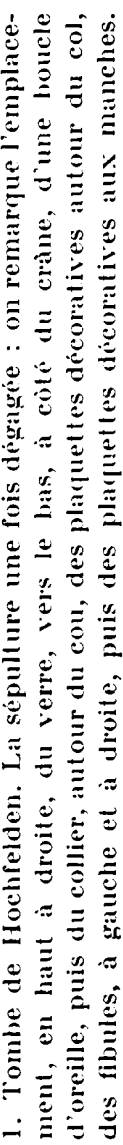




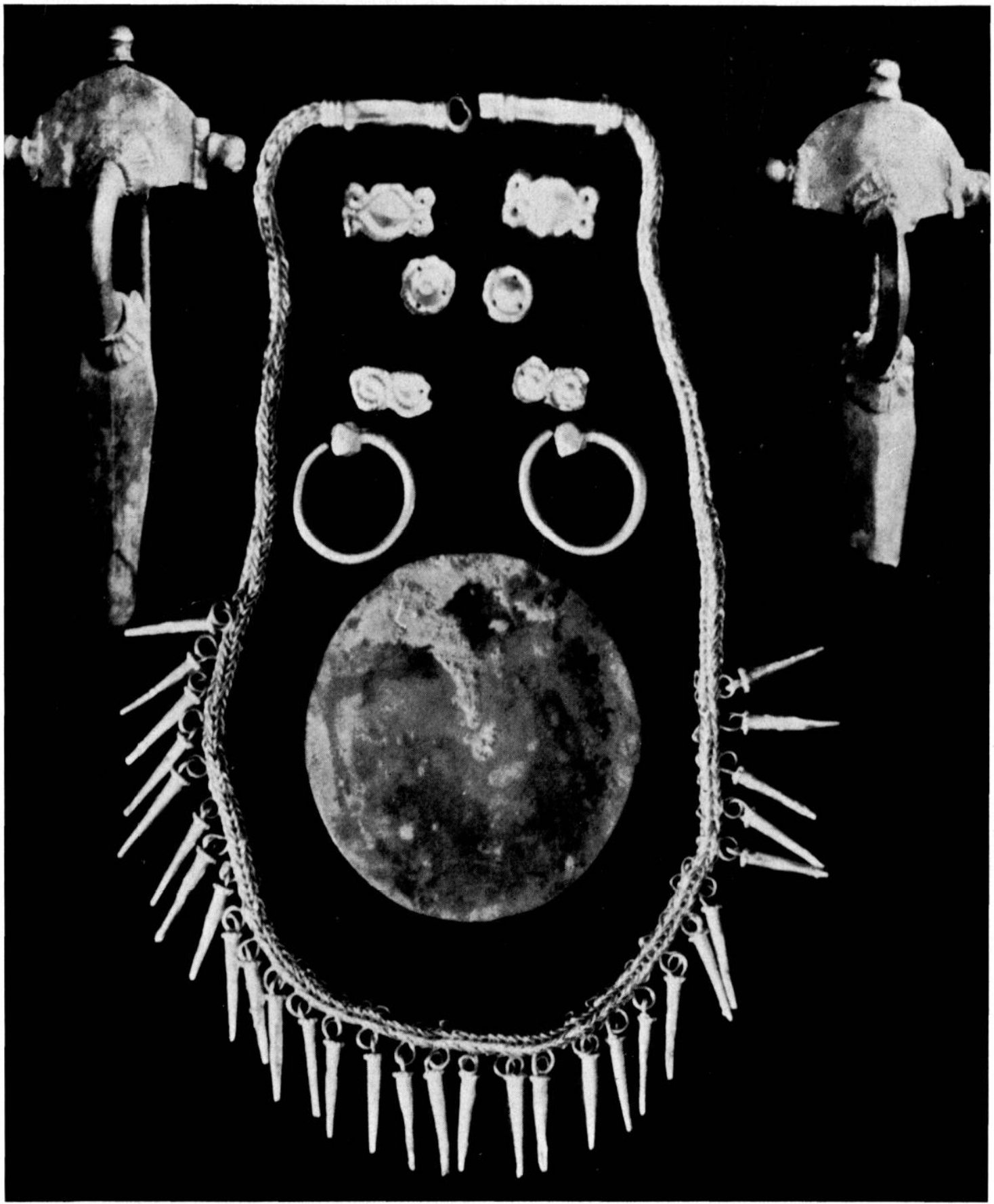

3. Parure de la tombe de IIochfelden.

bijoux de la basse époque romaine. Le travail de l'or est barbare et maladroit.

Le collier comprend une tresse d'or, terminée de chaque côté par un élément de fermoir, constitué d'une douille de section carrée, terminé par un anneau plat (fig. 1 et j). La longueur totale du collier est de $0,364 \mathrm{~m}$. Son poids de $27 \mathrm{gr}$. Trente pendeloques, constituées chacune d'une feuille d'or enroulée en forme de cône, décorée dans sa partie la plus large d'un anneau orné lui-même de grains au repoussé, imitant le grènetis, sont attachées à 


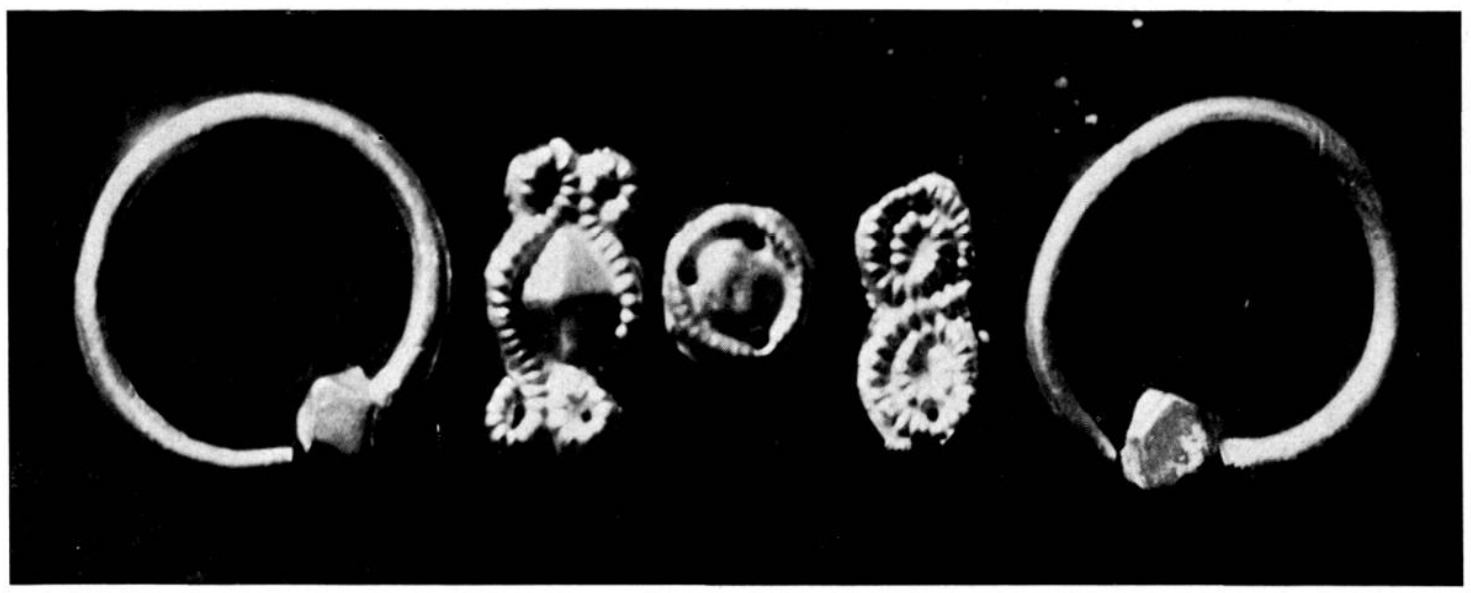

4. Tombe de Itochfelden. Boucles d'oreilles et plaquettes décoratives en or.

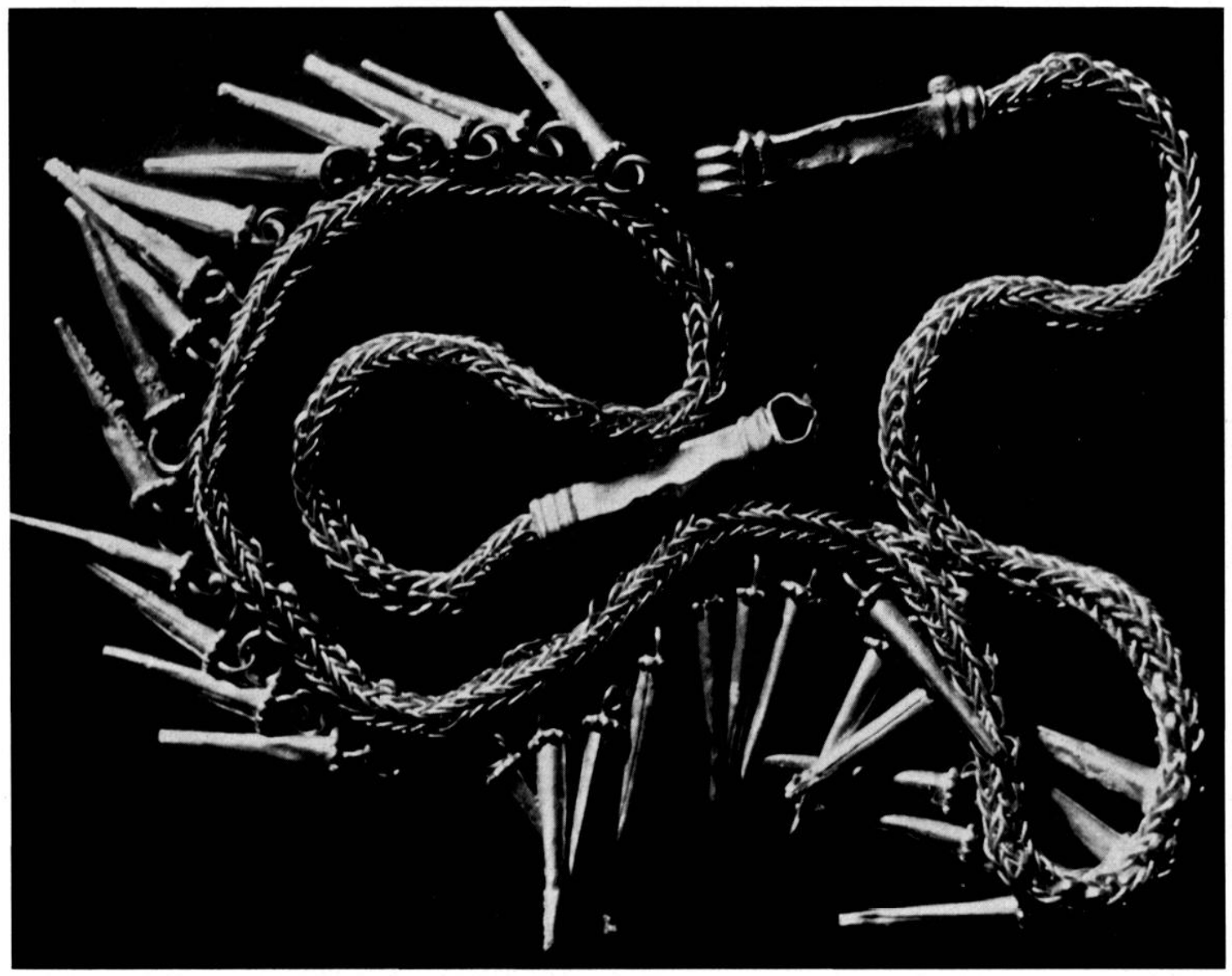

5. Tombe de Hochfelden. Collier d'or.

la tresse par deux fils d'or courbés en forme d'anneau, dont l'un est soudé ì l'intérieur du còne, el l'autre enfilé dans la tresse, ses extrémités étant retournées el aplaties. Comme pour les boucles d'oreilles, le travail d'orfieverie est assez grossier. Il s'agil de bijoux barbares, imitant probablement des modieles byzantins. Comme nous l'avons vu plus haul, le erochet. 


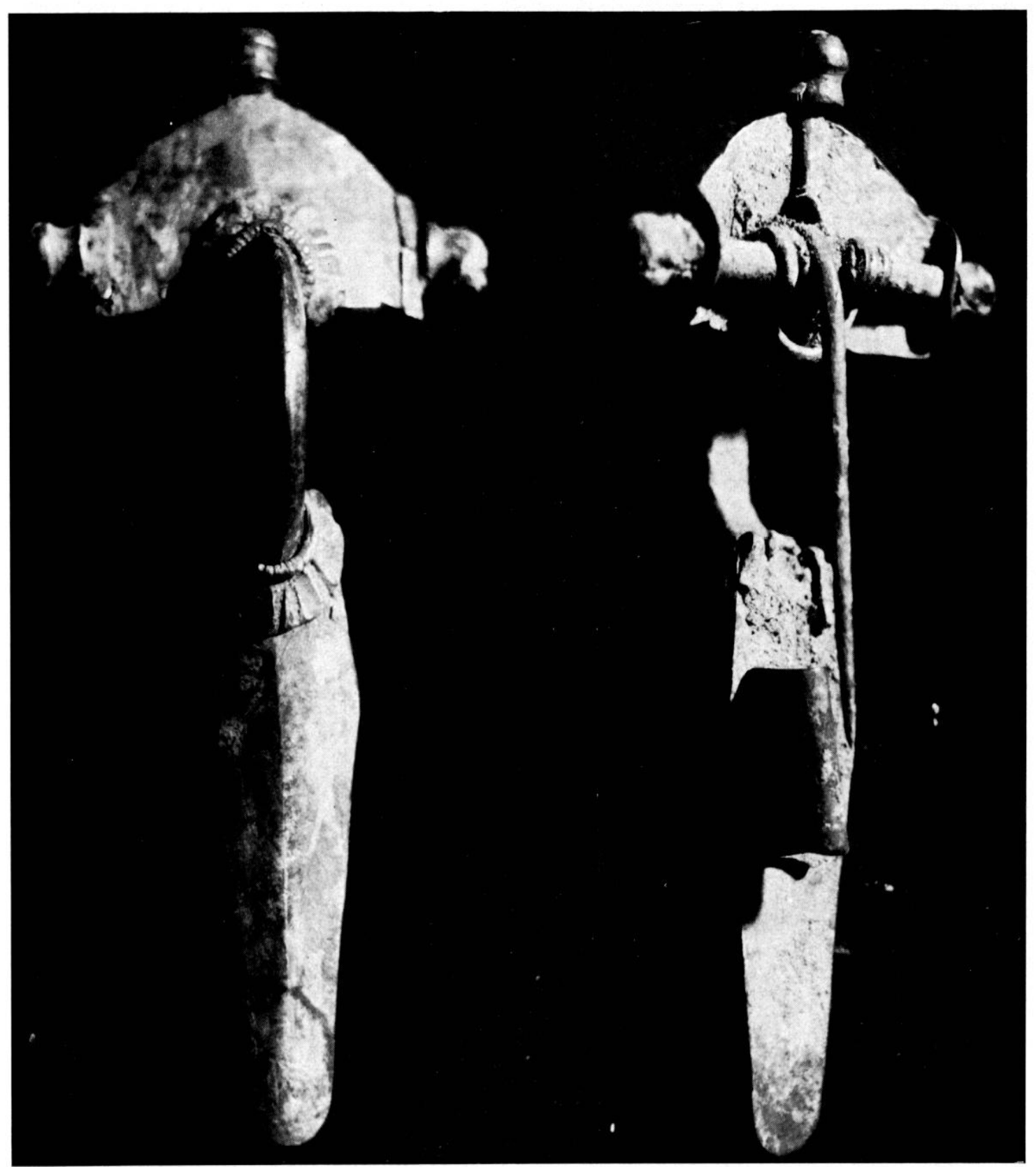

6 a et b. Tombe de Hochfelden. Fibule en argent, de type gothique.

de fermeture ne paraît pas avoir été déposé dans la tombe.

Les fibules, du type gothique, sont larges de $0,038 \mathrm{~m}$, longues de 0,082 . Elles comportent plusieurs éléments (fig. 6) : le corps même de la fibule, en argent, entièrement fondu d'une seule pièce, comprend la queue, l'anse et la tête, de forme semi-circulaire. Dans le milieu de l'axe horizontal, qui est recouvert d'une plaque d'argent enroulée en forme de cylindre, a été introduite de force une tige verticale de même métal, dont l'extrémitè est couverte par un bouton qui vient saillir au milieu de la tête de la fibule. Les deux extrémités de l'axe sont également terminées chacune par un bouton et sont fixées à la tête de la fibule par des plaques en argent doré. Sur l'axe vient s'enrouler un ressort en argent, dont une extrémité constitue la pointe de l'aiguille. Le porteagrafe, formé d'une plaque d'argent recourbée 


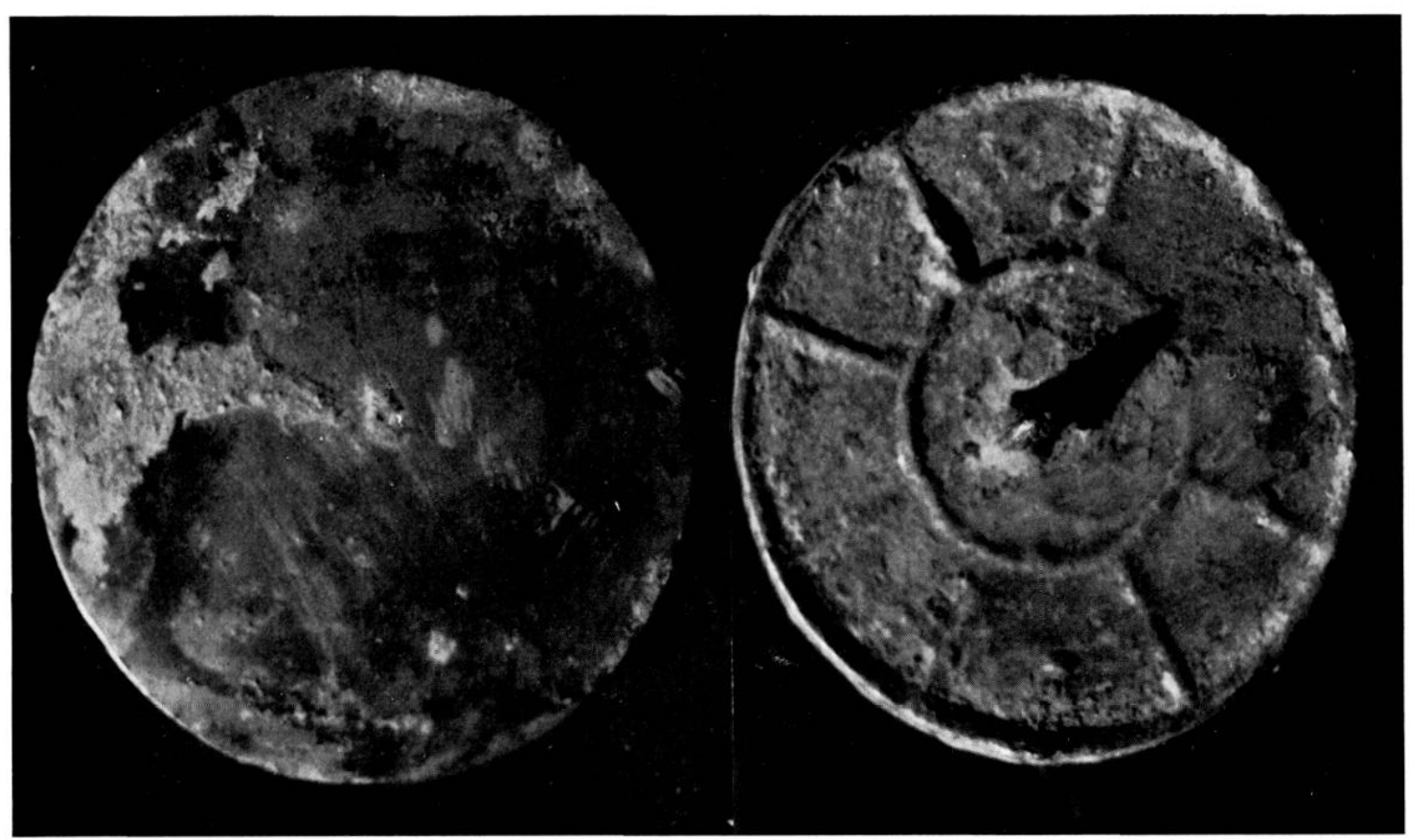

7. Tombe de Hochfelden. L.e miroir, face (à grauche) et revers.

d'un còté el aplatie de l'autre, était à l'origine soudé à la queue de la fibule mais il s'en est détaché. La jonction de l'anse à la tête et a la queue de la fibule est ornée d'une plaque d'argent dorée en forme de collerette gaufrée, agrafée par-dessous el couverte par dessus d'un anneau également en argent doré, orné de ciselures imitant grossièrement le grènelis.

Le miroir est constilué d'une plaque de bronze, d'un diamitre de $5,2 \mathrm{~cm}$ el d'une épaisseur, sur le bord, de $3 \mathrm{~mm}$ (fig. 7). L'envers est pourvu, en son milieu, d'une bélière el décoré de deux cercles concentriques et de huit rayons en relief, joignant ces cercles. La facture de la fonle est peu soignce et les barbes n'ont pas été limées. La face du miroir, très soigneusement polie, présente un reflet argenté.

Les plaquettes décoralives en or peuvent être divisées en trois catégories : les unes sont de forme circulaire et sont percées de trois trous, ce sont celles qui ornaient le col et les poignels ; les autres, qui se trouvaient à l'emplacement du coude, présentent lantòt une forme de losange et sont alors percées de quatre trous, tantôt une forme de double spirale et sont percées de deux trous. Les premières ont un diamètre moyen de $7 \mathrm{~mm}$, les secondes, une longueur de $15 \mathrm{~mm}$ et une largeur de $8 \mathrm{~mm}$, les dernières une longueur de $12 \mathrm{~mm}$ et une largeur de $6 \mathrm{~mm}$. Elles ont été embouties dans des matrices de fer ou de bronze, leur décor de demi-sphères au repoussé imitant celui du grènetis.

Ce groupement d'objets : fibules gothiques en argent, bijoux d'or pontiques imitant l'orfèvrerie byzantine, appartient spécifiquement au début du ve siècle. Relativement rares en Occident, les tombes de ce genre sont beaucoup plus fréquentes en IIongrie et sur les rives de la Mer Noire, qui semblent bien être leur palrie d'origine. Elles appartiennent, à proprement parler, à la période des grandes invasions (fin $d u v^{\mathbf{e}}$, début du ve siècle). La seule lombe contemporaine el présentant des caractères analogues qui ait été trouvée en Gaule est celle d'Airan, dans le Calvados, dont MM. Salin et France-Lanord ont repris récemment la publication et l'étude détaillée ${ }^{1}$. La sépulture d'Airan contenait deux très grandes fibules gothiques en argent du type du trésor

(1) Wonuments Piol, 43, p. 119 à 135 . 
de Szylagy Szömliö el décorées de cabochons sertis, une chainette d'or, des bijoux et des plaquettes très semblables à celles de Hochfelden.

D'après les travaux de Salin el de X. Aberg, les fibules gothiques peuvent être divisées en deux catégories : un type ancien, caractérisé par sa tête demi-circulaire, un type plus tardif, dont la tête présente à la base un rectangle, surmonté d'un arc de cercle. Le premier type appartient en propre au début du ve siècle, tandis que le second a duré jusqu'au $\mathrm{vi}^{\mathrm{e}}{ }^{2}$.

Si l'on se réfère à la carte de répartition des fibules gothiques, dressée par $\mathrm{N}$. $\mathrm{Aberg}^{3}$, on s'aperçoil que ce genre d'objets, rarissime dans la vallée du Rhin, n'y est connu qu'aux environs de Strasbourg. Il en existe naturellement un groupe important dans les vallées du Danube et de la Theisse, un autre groupe en Illyrie.

(2) X. Ant:R(, Frankien und Westgothen, p. 43 et suiv. (3) L. l., carte II.
Lin Gaule, nous remarquerons une série de fibules de ce genre dans la vallée de la Marne, une autre en Seine-Maritime, une autre dans le Limousin, des spécimens isolés en Charente et aux environs de Valence. Cette distribution doit-elle être mise en rapport avec des colonies de Sarmates ou d'Alains, installés à titre militaire par les Romains à la suite de la grande invasion de 406 ? La découverte en Alsace d'un ensemble de cette époque, venant se grouper avec la fibule gothique déjà connue des environs de Strasbourg, prouve que cette région était au cours des grandes invasions le point de passage obligé des émigrants venus de l'Est et désirant s'installer en Gaule. Ce fut d'ailleurs, un peu plus tard, la voie suivie par Attila en 451, puisque nous savons par ailleurs que les environs de Strasbourg ont également fourni les seuls objets appartenant à l'équipement des Huns qui aient été trouvés en Gaule, c'est la trouvaille de Mundolsheim.

J.-J. HATT. 